\title{
A GENERALIZATION OF PELLET'S THEOREM CONCERNING THE ZEROS OF A POLYNOMIAL ${ }^{1}$
}

BERNARD W. LEVINGER

1. Introduction. Let $f(z)=a_{0}+a_{1} z+\cdots+a_{n} z^{n}$ be a polynomial of degree $n$ with complex coefficients. Many useful estimates for the zeros of $f(z)$ depend only upon the moduli, $\left|a_{k}\right|$, of some or all of the coefficients. As such, these estimates apply not only to $f$ but to certain classes $\mathfrak{F}_{A}$ of polynomials related to $f$ which will be defined below.

We have discovered a simple characterization of the set of all zeros of polynomials in such a class $\mathfrak{F}_{\boldsymbol{A}}$ by a system of algebraic inequalities. This is an extension of well known theorems by Pellet and Walsh. Our results are sharp for each class $\mathcal{F}_{A}$. Thus, they can be used to estimate to what extent a given bound for the zeros of $f(z)$ is best possible. We illustrate this by using our main theorem to give generalizations of theorems by Marden and Ostrowski.

Our main point is that, by applying quite elementary methods, one can obtain a number of theorems related to Pellet's theorem. These theorems depend upon the existence of disjoint regions in the complex plane containing the zeros of all polynomials in a class $\mathfrak{F}_{\boldsymbol{A}}$. Although the inequalities in Theorem 1 may be complicated, they should prove useful for the derivation of simpler estimates and also for the determination of how close a known estimate is to the best possible result. Our results are related to estimates for the characteristic values of a matrix using minimal Gerschgorin sets due to R. S. Varga [10], [1], in the sense that we prove theorems about the class $\mathcal{F}_{A}$ rather than the individual polynomial $f(z)$.

Our main theorem is proved in $\$ 2$ and applications are given in $\S 3$. The author would like to thank Professor Varga for helpful discussions.

2. The main result. Let $f(z)=a_{0}+a_{1} z+\cdots+a_{n} z^{n}$ be an arbitrary polynomial of degree $n$ with complex coefficients and $A$ a subset of $\{0,1, \cdots, n\}$. We define a class of polynomials

$$
\begin{aligned}
& \mathfrak{F}_{A}(f)=\left\{q(z)=\sum_{k=0}^{n} b_{k} z^{k}|| b_{k}|=| a_{k} \mid, k=0, \cdots, n ;\right. \\
&\left.b_{i}=a_{i}, \quad i \in A\right\} .
\end{aligned}
$$

1 Presented to the Society, January 29, 1965 under the title On the location of zeros of polynomials; received by the editors November 5, 1966. 
(In general, we shall write $\mathcal{F}_{A}$ for $\mathcal{F}_{A}(f)$ when the context is clear.)

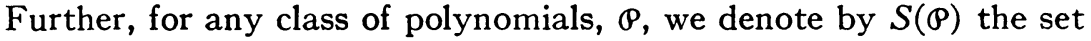
of zeros,

$$
S(\odot)=\{\sigma \mid q(\sigma)=0 \text { for some } q \in \odot\} .
$$

With these definitions we can state our main result which precisely characterizes $S\left(\mathfrak{F}_{A}\right)$ by a system of algebraic inequalities.

TheOREM 1. Let $f(z)=\sum_{i=0}^{n} a_{j} z^{j}$ be a polynomial of degree $n$ with complex coefficients and $A$ a subset of $\{0,1, \cdots, n\}$. Then

(1) $A$ complex number $\sigma$ is a zero of a polynomial $q(z) \in F_{A}(f)$ if and only if, for each $k \notin A$,

$$
2\left|a_{k}\right||\sigma|^{k}-\sum_{j \notin A}\left|a_{j}\right||\sigma|^{j} \leqq\left|\sum_{i \in A} a_{i} \sigma^{i}\right| \leqq \sum_{j \notin A}\left|a_{j}\right||\sigma|^{j}
$$

(2) If $k \notin A$ and, for all points $z$ on a simple closed curve $C$ surrounding the origin,

$$
2\left|a_{k}\right|\left|z^{k}\right|>\sum_{j \notin A}\left|a_{j}\right||z|^{j}+\left|\sum_{i \in A} a_{i} z^{i}\right|,
$$

then each $q(z) \in \mathcal{F}_{A}(f)$ has exactly $k$ zeros in the bounded region enclosed by $C$.

(3) If

$$
\left|\sum_{i \in A} a_{i} z^{i}\right|>\sum_{j \notin A}\left|a_{j}\right||z|^{j}
$$

on a simple closed curve $C^{\prime}$, then each $q(z) \in \mathcal{F}_{A}(f)$ has the same number of zeros in the bounded region $D^{\prime}$ enclosed by $C^{\prime}$. This number is the number of zeros in $D^{\prime}$ of $\sum_{i \in A} a_{i} z^{i}$.

The proof follows by Rouche's theorem and the following lemma.

LeMmA 1. Let $\alpha_{1}, \cdots, \alpha_{N}$ be nonnegative real numbers and $\rho$ an arbitrary complex number. Then there exist real numbers $\theta_{1}, \cdots, \theta_{N}$ such that

$$
\rho=\sum_{k=1}^{N} \alpha_{k} e^{i \theta_{k}}
$$

if and only if

$$
2 \max _{1 \leqq j \leqq N} \alpha_{j}-\sum_{k=1}^{N} \alpha_{k} \leqq|\rho| \leqq \sum_{k=1}^{N} \alpha_{k}
$$


Proof. (2.5) is equivalent to the statement that there is a closed polygon in the complex plane with sides $\alpha_{1}, \cdots, \alpha_{N},|\rho|$. It is possible to construct such a polygon if and only if the length of any side does not exceed the sum of the lengths of the other sides. This condition is equivalent to (2.6).

PROOF OF THEOREM 1. If $\sigma$ is a zero of a polynomial $q(z)$ in $\mathfrak{F}_{A}(f)$ then $q(\sigma)=\sum_{j=0}^{n} b_{j} \sigma^{j}=0$, or, from (2.1),

$$
\sum_{i \in A} a_{i} \sigma^{i}=-\sum_{j \notin \Delta} b_{j} \sigma^{j}
$$

This corresponds to (2.5) with $\rho=\sum_{i \in A} a_{i} \sigma^{i}$ and $\alpha_{k}=\left|b_{j} \sigma^{j}\right|=\left|a_{j}\right||\sigma|^{j}$. Hence, by Lemma 1, (2.7) can hold if and only if the analogue of (2.6) holds,

$$
2 \max _{k \notin A}\left|a_{k}\right||\sigma|^{k}-\sum_{j \notin A}\left|a_{j}\right||\sigma|^{j} \leqq\left|\sum_{i \in A} a_{i} \sigma^{i}\right| \leqq \sum_{j \notin A}\left|a_{j}\right||\sigma|^{j},
$$

which is equivalent to (2.3) for each $k \notin A$. This completes the proof of part (1) of the theorem. (2) and (3) follow immediately from Rouche's theorem.

For the applications, it is useful to introduce some notation. Let

$$
\begin{aligned}
& h_{k, \Lambda}(f ; \sigma)=(-1)^{\ell_{k}, A}\left|\sum_{i \in A} a_{i} \sigma^{i}\right|+\sum_{j \notin A}(-1)^{\delta_{j}, k}\left|a_{j}\right||\sigma|^{j}, \\
& 0 \leqq k \leqq n,
\end{aligned}
$$

where $\epsilon_{k, A}=\sum_{i \in A} \delta_{i, k}$ and $\delta_{i, k}$ is the Kronecker delta, and

$$
C_{k, A}(f)=\left\{z \mid h_{k, A}(f ; z) \geqq 0\right\} .
$$

Then Theorem 1 states that

$$
S\left(F_{A}\right)=\bigcap_{k=0}^{n} C_{k, A}(f)=\left\{z \mid h_{k, A}(f ; z) \geqq 0,0 \leqq k \leqq n\right\} .
$$

We now show that $C_{k, A}(f)$ can be characterized as the set of zeros of a class of polynomials.

TheOREM 2. For $k \notin A$, let

$$
\mathcal{F}_{k, A}(f)=\left\{q(z)=\sum_{j=0}^{n} b_{j} z^{j}\left|b_{i}=a_{i}, i \in A ;\right| b_{k}|\geqq| a_{k} \mid ;\right.
$$

and for $k \in A$, let

$$
\left.\left|b_{j}\right| \leqq\left|a_{j}\right|, j \neq k, j \notin A\right\}
$$




$$
\begin{aligned}
& \mathscr{F}_{k, A}(f)=\left\{q(z)=\sum_{j=0}^{n} b_{j} z^{j}\left|b_{i}=\lambda a_{i},\right| \lambda \mid \geqq 1, i \in A\right. \\
&\left.\left|b_{j}\right| \leqq\left|a_{j}\right|, j \in A\right\} .
\end{aligned}
$$

Then $S\left(\mathfrak{F}_{k, A}(f)\right)=C_{k, A}(f), k=0,1, \cdots, n$.

Proof. If $q(z) \in \mathcal{F}_{k, A}(f)$, it follows from the definitions that $h_{k, A}(q ; z) \leqq h_{k, A}(f ; z)$ for all $z$, and hence that $C_{k, A}(q) \subset C_{k, A}(f)$. By (2.10), $S\left(\mathfrak{F}_{A}(q)\right) \subset C_{k, A}(q)$ and thus $S\left(\mathfrak{F}_{k, A}(f)\right) \subset C_{k, A}(f)$.

For the converse, suppose that $h_{k, A}(f ; \sigma) \geqq 0$. We can then construct a polynomial $q(z) \in \mathcal{F}_{k, A}(f)$ for which $h_{k, A}(q ; \sigma)=0$. Then it follows from (2.8) that $h_{j, A}(q ; \sigma) \geqq 0$ for $j=0,1, \cdots, n$ and hence, by (2.10), that $\sigma \in S\left(F_{A}(q)\right) \subset S\left(\mathscr{F}_{k, A}(f)\right)$. Thus $C_{k, A}(f) \subset S\left(\mathscr{F}_{k, A}(f)\right)$ which completes the proof.

We illustrate the construction of $q(z)$ for the case that $k \in A$ and $h_{k, A}(f ; \sigma)=\sum_{j \notin A}\left|a_{j}\right||\sigma|^{j}-\left|\sum_{i \in A} a_{i} \sigma^{i}\right|>0$. Then

$$
\lambda=\left|\sum_{i \in A} a_{i} \sigma^{i}\right| / \sum_{j \notin A}\left|a_{j}\right||\sigma|^{j}<1,
$$

and $q(z)=\sum_{i \in A} a_{i} z^{i}+\lambda \sum_{j \notin A} a_{j} z^{j} \quad$ is $\quad$ in $\mathfrak{F}_{k, A}(f)$ and satisfies $h_{k, A}(q ; \sigma)=0$. A similar construction works if $k \notin A$.

We remark that the use of Rouché's theorem could have been avoided in the proof of the second part of Theorem 1. These statements also follow quite easily from Theorem 2 and the continuous dependence of the zeros of a polynomial on the coefficients [11].

3. Application. We will first examine the consequences of Theorem 1 , when $A=\varnothing$, the empty set, and $A=(m, p)$, a set of two indices. In the former case, our theorem reduces to a theorem of Pellet and its converse due to Walsh. In the latter case we obtain a refinement of a theorem of Marden. We will conclude with a problem related to Pellet's theorem which was studied by Parodi and Ostrowski [5]-[8].

(1) $A=\varnothing$. In this case we shall write (2.8) as

$$
h_{k}(z) \equiv h_{k, \phi}(f ; z)=\sum_{j=0}^{n}(-1)^{\delta_{j, k}}\left|a_{j}\right||z|^{j}
$$

Since $h_{k}(z)=h_{k}(|z|)$, the regions $C_{k, \phi}(f),(2.9)$, considered in $\$ 2$ will have circular boundaries.

A classical theorem of Pellet [3, Theorem 28,1] states that if $h_{k}(z)$ has two positive zeros, $0<x_{1}<x_{2}$, then $f(z)$ has exactly $k$ zeros in $|z| \leqq x_{1}$. Walsh [4], [11], [3, Theorem 28,2] proved the following converse: If $\rho$ is a positive real number such that each $q(z) \in \mathscr{F}_{\phi}(f)$ has exactly $k$ zeros in $|z| \leqq \rho$, but $\rho \notin S\left(\mathcal{F}_{\phi}\right)$, then $h_{k}(\rho)<0$. Pellet's the- 
orem is an immediate consequence of Theorem 1 , since, if $h_{k}(z)$ has two positive zeros, $h_{k}(z)<0$ for $x_{1}<|z|<x_{2}$. Walsh's theorem follows from the fact that $h_{j}(|z|)=h_{j}(z)$ for all $j=0,1, \cdots, n$. Thus, if $\rho \notin S\left(F_{\phi}\right), h_{j}(\rho)<0$ for some $j, 0 \leqq j \leqq n$. Hence by Theorem 1 , each $q(z) \in \mathcal{F}_{\phi}$ has exactly $j$ zeros in $|z| \leqq \rho$, and thus $j=k$.

Walsh also proved a second theorem for the limiting case of Pellet's theorem in which $h_{k}(z)$ has a double root $\rho[11],[3$, Theorem 28, 3]. In this case, $h_{k}(z) \geqq 0$, for all $z$ and Theorem 1 gives no information. However, if $q(z)=\sum_{i=0}^{n} b_{i} z^{i}$ is in $\mathfrak{F}_{k, \phi}$ with $\left|b_{k}\right|>\left|a_{k}\right|$, then $h_{k, \phi}(q ; \rho)<0$ and thus $q(z)$ has $k$ zeros in $|z| \leqq \rho$. Since $f(z)$ is the limit of a sequence of such $q(z)$ and the zeros of a polynomial are continuous functions of the coefficients, it follows that $f(z)$ also has at least $k$ zeros in $|z| \leqq \rho$. A refinement of the argument shows that if $f(z)$ has $\delta$ zeros on $|z|=\rho$, these are double zeros and $f(z)$ has $k-\delta$ zeros in $|z|<\rho$.

(2) $A=(m, p)$ where $0 \leqq m<p \leqq n, a_{m} a_{p} \neq 0$. For this case, Marden [2], [3, Theorem 29, 1] proved a useful refinement of Pellet's theorem:

Let $G\left(r_{0}, r ; p, \alpha\right)$ denote the boundary of the gear wheel shaped region formed by adding to the points of $|z| \leqq r_{0}$ those points of $r_{0} \leqq|z| \leqq r$ which satisfy $\alpha+(4 k-3) \pi / 2 \leqq p$ arg $z \leqq \alpha+(4 k-1) \pi / 2$, $k=1,2, \cdots, p$. Marden's theorem essentially states that if $h_{p}(z)$ has two positive zeros $r<R$, then $\Psi_{m}(z)=h_{p}(z)-\left|a_{m}\right||z|^{m}$ also has two positive zeros $r_{m}<r<R<R_{m}$ and $f(z)$ has exactly $p$ zeros in the closed region bounded by $G\left(r_{m}, r ; p-m, \alpha_{m}\right)$, where $\alpha_{m}=\arg a_{m} / a_{p}$, and no zeros in the annular region between $G\left(r_{m}, r ; p-m, \alpha_{m}\right)$ and $G\left(R, R_{m} ; p-m, \alpha_{m}+\pi\right)$.

This is a theorem about the class $\mathcal{F}_{(m, p)}(f)$, because the result depends upon the value of $\alpha_{m}=\arg a_{m} / a_{p}$. It is a simple consequence of Theorem 1. The statements about the zeros of $\Psi_{m}(z)$ follow from $\Psi_{m}(r)<0, \Psi_{m}(R)<0$, and, in order to prove the theorem, it suffices to show that $h_{p,(m, p)}(z)=\sum_{j \neq m, p}\left|a_{j}\right||z|^{i}-\left|a_{m} z^{m}+a_{p} z^{p}\right|<0$ in the annular region. For $r<|z|<R$, this follows immediately from the fact that $h_{p,(m, p)}(z) \leqq h_{p}(z)<0$. For $\alpha_{m}+(4 k-1) \pi / 2 \leqq(p-m)$ arg $z \leqq \alpha_{m}$ $+(4 k+1) \pi / 2$, we have $\left|a_{m} z^{m}+a_{p} z^{p}\right| \geqq\left(\left|a_{m} z^{m}\right|^{2}+\left|a_{p} z^{p}\right|^{2}\right)^{1 / 2}>\left|a_{p} z^{p}\right|$ when $|z|>0$. Thus in this sector, $h_{p,(m, p)}(z)<\Psi_{m}(z)<0$, when $r_{m} \leqq|z|<R_{m}$.

Theorem 1 actually gives an improvement of Marden's result, since it yields the precise boundaries of $S\left(F_{(m, p)}\right)$. We illustrate this with a simple example. If $f(x)=1+2 x+4 x^{2}+x^{3}$, then Pellet's theorem shows that

$$
S\left(\Im_{\phi}(f)\right)=\{0.303 \leqq|z| \leqq 1\} \cup\{3.3 \leqq|z| \leqq 4.5\} .
$$


Marden's result shows that $S\left(F_{(0,2)}(f)\right)$ lies in the domain formed from $S\left(\mathcal{F}_{\phi}\right)$ by removing

$$
\begin{aligned}
\{z \mid-\pi / 4 & \text { arg } \left.z \leqq \pi / 4,2-2^{1 / 2} \leqq|z| \leqq 2+2^{1 / 2}\right\} \\
& \cup\left\{z\left|3 \pi / 4 \leqq \arg z \leqq 5 \pi / 4,2-2^{1 / 2} \leqq\right| z \mid \leqq 2+2^{1 / 2}\right\} .
\end{aligned}
$$

However, from Theorem 1, we find that $S\left(F_{(0,2)}(f)\right)$ actually has three disjoint components, as illustrated in the figure.

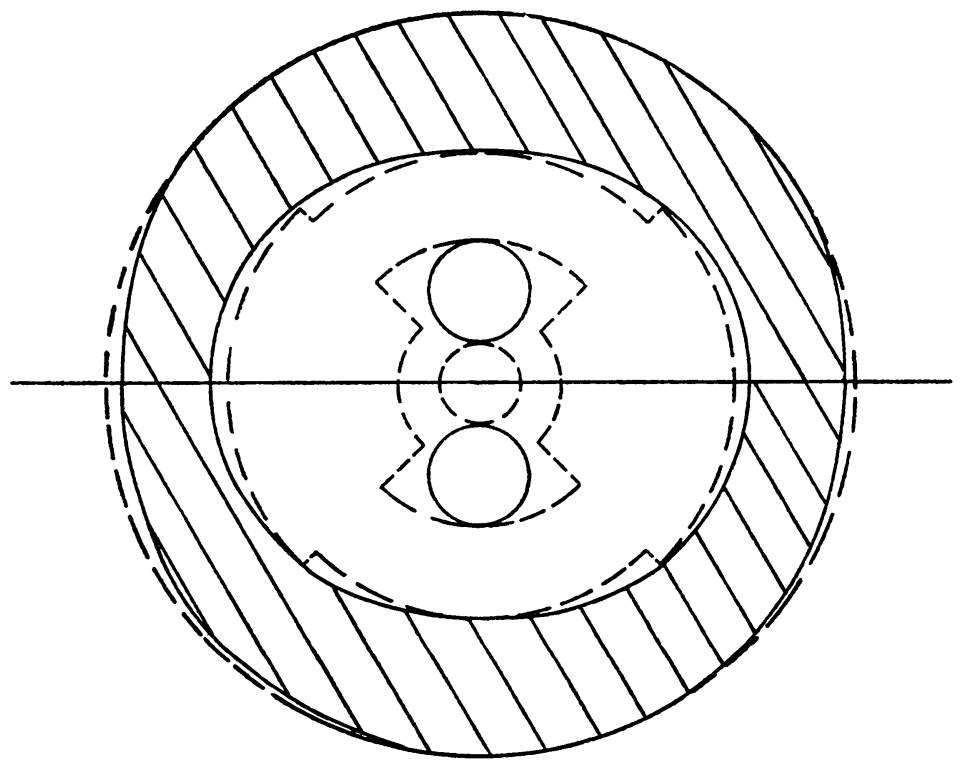

$S\left(\mathcal{F}_{(0.2)}\left(1+2 x+4 x^{2}+x^{3}\right)\right.$ ) (not to scale). Marden's estimate [2] indicated by the broken line.

We remark that, since $h_{p,(m, p)}(z)=\min \left(h_{m}(z), h_{p}(z)\right)$ when $\arg z=\alpha_{m}$ $+\pi / p-m$, and $h_{k,(m, p)}(z)=h_{k}(z)$ when $\arg z=\alpha_{m}$ and $k \neq m, p, S\left(\mathcal{F}_{(m, p)}\right)$ has more than one connected component if and only if the same holds for $S\left(\mathcal{F}_{\phi}\right)$. Thus, if $\sigma \in S\left(\mathcal{F}_{\phi}\right)$, one can always find a $\sigma^{\prime} \in S\left(\mathcal{F}_{(m, p)}\right)$ with $\left|\sigma^{\prime}\right|=|\sigma|$. This shows that, if one wants to obtain bounds for the zeros of a polynomial which are significantly stronger than those derived from Pellet's theorem, one should fix at least three coefficients.

Specht [9] has given some estimates for $S\left(F_{A}\right)$ when $A=(0,1$, $\cdots, k, n), k<n$. These are also easy consequences of Theorem 1 . However, it appears that $S\left(\mathfrak{F}_{A}\right)$ cannot easily be computed by hand if $A$ contains more than two indices. 
(3) A problem related to Pellet's theorem was studied by Parodi and Ostrowski [5]-[8]. If the coefficients $a_{0}, \cdots, a_{n-2}$ of $f(z)$ are sufficiently small compared to $a_{n-1}$, then one can find two disjoint disks $\left|a_{n} z+a_{n-1}\right| \leqq \rho$ and $|z| \leqq \rho^{*}$ which contain exactly one and $(n-1)$ zeros of $f(z)$, respectively. Ostrowski gave necessary and sufficient conditions on the coefficients for the existence of such $\rho$ and $\rho *$. With little extra work we shall solve a more general problem.

Let $A$ be a subset of $\{0,1, \cdots, n\}$ which excludes at least two numbers, and let $r$ and $s$ be respectively the smallest and largest integers not in $A, 0 \leqq r<s \leqq n$. We define

$$
\begin{aligned}
& U_{A}=\sum_{j \notin A}\left|a_{j}\right| \\
& \Phi_{A}=\left\{q(z)=\sum_{j=0}^{n} b_{j} z^{j}\left|b_{j}=a_{j}, j \in A ; \sum_{k \notin A}\right| b_{k} \mid=U_{\Delta}\right\} .
\end{aligned}
$$

Theorem 3. $\sigma \in S\left(\Phi_{A}\right)$ if and only if

$$
\left|\sum_{i \in A} a_{i} \sigma^{i}\right| \leqq U_{A} \max \left(|\sigma| r,|\sigma|^{s}\right) .
$$

Proof. If $\sigma \in S\left(\Phi_{A}\right)$, there is a $q(z) \in \Phi_{A}$ with $q(\sigma)=\sum_{j=0}^{n} b_{j} \sigma^{j}=0$. Thus, $\sigma \in S\left(\mathcal{F}_{A}(q)\right)$ and by (2.3) and (3.3)

$$
\begin{aligned}
\left|\sum_{i \in A} a_{i} \sigma^{i}\right| & \leqq \sum_{j \notin A}\left|b_{j}\right||\sigma|^{j} \\
& \leqq \sum_{j \notin A}\left|b_{j}\right| \max _{j \notin A}|\sigma|^{j}=U_{A} \max \left(|\sigma|^{r},|\sigma|^{\circ}\right) .
\end{aligned}
$$

Conversely, if (3.4) holds, we can find

$$
g(z)=\sum_{i \in A} a_{i} z^{i}+U_{A}\left(\beta z^{r}+(1-\beta) z^{z}\right) \text { in } \Phi_{A},
$$

for which $h_{k, A}(g ; \sigma) \geqq 0,0 \leqq k \leqq n$, so that $\sigma \in S\left(F_{A}(g)\right) \subset S\left(\Phi_{A}\right)$ by (2.10). (3.4) is equivalent to

$$
\theta=\left|\sum_{i \in A} a_{i} \sigma^{i}\right| /\left(U_{A} \max (|\sigma| r,|\sigma| s)\right) \leqq 1 .
$$

If $|\sigma| \leqq 1$, then (3.5) gives $\left|\sum_{i \in A} a_{i} \sigma^{i}\right|=\theta U_{A}|\sigma|^{r}$, and it is easily verified that $\beta=\theta$, for $1 \geqq \theta \geqq|\sigma| * /\left(|\sigma|^{r}+|\sigma|^{*}\right)$, and $\beta=|\sigma| * /\left(|\sigma|^{*}\right.$ $+|\sigma| r)$, for $|\sigma| \cdot /\left(|\sigma| r+|\sigma|^{\circ}\right) \geqq \theta \geqq 0$, give the desired result. An analogous choice works for $|\sigma| \geqq 1$, and this completes the proof.

Finally, we state the analogue of Ostrowski's necessary and sufficient conditions for the case when $A$ consists of two indices. 
Theorem 4. Let $A=\{m, p\}$ with $0<m<p \leqq n$. Then $S\left(\Phi_{A}\right)$ has at least two disjoint components if and only if at least one of the conditions, I and II, I and II', III, is satisfied.

I. $\left|a_{m} / p\right|^{p}\left|a_{p} / m\right|^{-m}>\left[U_{A} /(p-m)\right]^{(p-m)}$.

II. $x_{1}<1$, where $x_{1}$ is the least positive root of $\left|a_{p}\right| x^{p}-\left|a_{m}\right| x^{m}+U_{A}$ $=0$.

II'. $p=n=m+1,\left|a_{n}\right|+U_{A} \geqq\left|a_{n-1}\right|>2 \max \left(\left|a_{n}\right|,\left|a_{n} U_{A}\right|^{1 / 2}\right)$, and $x_{1} \geqq 1$, where $x_{1}$ is as in II.

III. $n>p>m$,

$$
\left|a_{p} /(n-m)\right|^{(n-m)}\left|a_{m} /(n-p)\right|^{(p-n)}>\left[U_{A} /(p-m)\right]^{(p-m)},
$$

and $x_{1}^{\prime}<1$, where $x_{1}^{\prime}$ is the least positive root of $\left|a_{m}\right| x^{(n-m)}-\left|a_{p}\right| x^{(n-p)}$ $+U_{A}=0$.

This theorem follows from Theorem 3 by an examination of various cases. We will omit the proof which is not hard but somewhat lengthy.

\section{REFERENCES}

1. B. W. Levinger and R. S. Varga, Minimal Gerschgorin sets. II, Pacific J. Math. 17 (1966), 199-210.

2. M. Marden, A refinement of Pellet's theorem, Bull. Amer. Math. Soc. 54 (1948), 550-57.

3. M. Marden, The geometry of zeros of a polynomial in a complex variable, Math. Surveys No. 3, Amer. Math. Soc., Providence, R. I., 1966.

4. A. Ostrowski, On a theorem by J. L. Walsh concerning the moduli of roots of algebraic equations, Bull. Amer. Math. Soc. 47 (1941), 742-746.

5. A. Ostrowski, Über einige Sätze von Herrn M. Parodi, Math. Nachr. 19 (1958), $331-338$.

6. R. J. Painter, Extensions of theorems of Ostrowski on the zeros of polynomials, J. Elisha Mitchell Sci. Soc. 80 (1964), 45-48.

7. M. Parodi, Sur deux propriêtés des polynomes, C. R. Acad. Sci. Paris 242 (1956), 598-600.

8. M. Parodi, Sur la localisation des zéros des polynonzes, C. R. Acad. Sci. Paris 243 (1956), 1093-96.

9. W. Specht, Zur Werteverteilung der Polynome, J. Reine Angew. Math. 212 (1963), 73-79.

10. R. S. Varga, Minimal Gerschgorin sets, Pacific J. Math. 15 (1965), 719-729.

11. J. L. Walsh, On Pellet's theorem concerning the roots of a polynomial, Ann. of Math. (2) 26 (1924), 59-64.

Case Institute of Technology 